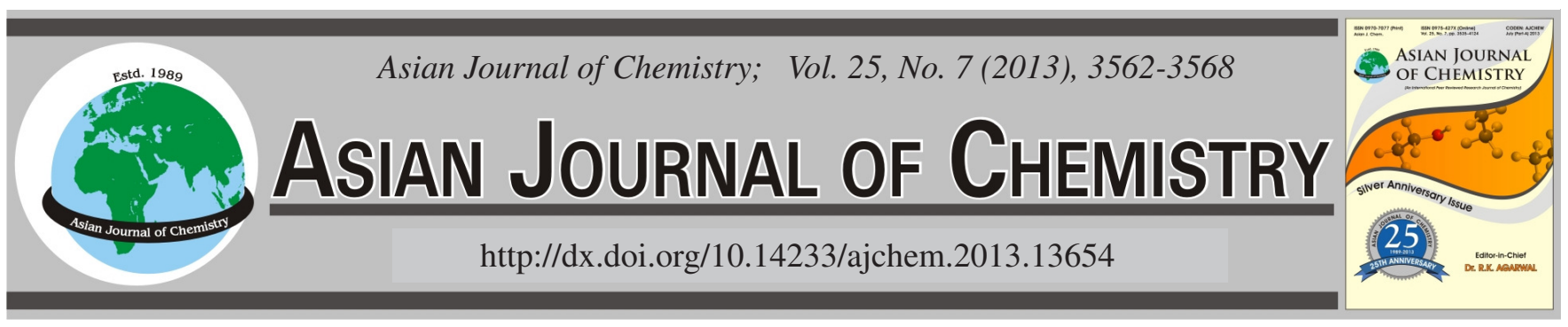

\title{
Preparation of Magnetic Dextran-Mitomycin C Prodrug Conjugate and Its in vivo Antitumor Activity
}

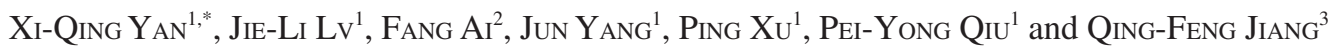

${ }^{1}$ College of Pharmacy, Xinxiang Medical University, Xinxiang 453003, P.R. China

${ }^{2}$ Department of Pediatric Surgery, First Affiliated Hospital of Xinxiang Medical University, Weihui 453100, P.R. China

${ }^{3}$ Nuokai Biological Pharmaceutical Co. Ltd., Changsha 410008, Hunan Province, P.R. China

*Corresponding author: Tel/Fax: +86 37 33831682; E-mail: yxqdoctor@163.com

(Received: 27 February 2012;

Accepted: 4 January 2013)

AJC-12649

\begin{abstract}
As a broad spectrum and strong cytotoxicity against human neoplastic diseases, mitomycin C (MMC)'s clinical use was greatly restricted for its indiscriminate action on tumor and normal cell. This paper proposed the work to devise magnetic targeted mitomycin $\mathrm{C}$ conjugate nanoparticles by arbodiimide-catalyzed reaction, so as to the mitomycin $\mathrm{C}$ could be release mostly in targeted issues. The properties of magnetic fluid (MF), dextran encapsulated magnetic (DM) and mitomycin C conjugate nanoparticles (MMC-DM) were characterized by Fourier transform infrared, X-ray diffraction, transmission electron microscope, laser particle analyzer and magnetometer. The drug release experiment was conducted at $37^{\circ} \mathrm{C}$ in phosphate buffer solution $(\mathrm{pH} \mathrm{7.4)} \mathrm{on} \mathrm{dissolution.} \mathrm{The} \mathrm{grafting} \mathrm{ratio} \mathrm{and} \mathrm{drug} \mathrm{loading} \mathrm{were}$ determined by gel chromatograph spectrophotometry. The in vivo antitumor experiment was carried out in BACA/C nude mice. The results showed that (1) the average diameter of the magnetic conjugate nanoparticles was $c a .125 \mathrm{~nm},(2)$ the mitomycin C in the MMCDM nanoparticles exhibited extended release in vitro release experiment and (3) the obvious inhibition of the growth of tumor implied that the active ingredient in the magnetic conjugate could be led to the aimed site and decrease damage to normal issues.
\end{abstract}

Key Words: Magnetic, Dextran, Nanoparticles, Mitomycin C, Conjugate.

\section{INTRODUCTION}

The selective delivery of chemotherapeutic agents to their site of action should increase therapeutic effectiveness but decrease side effects ${ }^{1}$. However, cancer chemotherapy with cytotoxic ingredients is often hampered by their indiscriminate action on both tumor and normal cells. To avoid these adverse effects, an ideal possible way is to concentrate their cytotoxicity on the tumor site by changing their chemical or biological properties. Such methods have been constantly "updated" including liposomes, nanoparticles, special technology polymers and virus, etc. ${ }^{2}$.

Polymer conjugates are becoming established as a new approach towards improved cancer therapy ${ }^{3,4}$. These watersoluble, hybrid constructs fall into two main categories: polymer-protein conjugates and polymer-drug conjugates. Polymer-drug conjugation can alter the biodistribution, elimination and rate of metabolism of covalently bound drugs 5 . The parent agents of these conjugates can be acted as molecular reservoir form while reducing acute toxic and immunosuppressive effects towards the host yet maintaining local therapeutic potency.
Dextrans serve as one of the most promising macromolecular carrier candidates for a wide variety of therapeutic agents due to their excellent physico-chemical properties and physiological acceptance ${ }^{6}$. The dextran conjugates can either be irreversibly linked or reversibly linked. Reversible dextran derivatives with conjugates include dextran esters, dextran ethers and dextran amides.

Mitomycin $\mathrm{C}$ is a bifunctional alkylating agent, having the enhanced activity in hypoxic environments and a potential for locoregional treatment of solid tumors since a significant percentage of viable cancer cells within a solid tumor can be hypoxic ${ }^{7}$. However, use of MMC is associated with a number of acute and chronic toxicities, such as irreversible myelosuppression and hemolyticuremic syndrome, which limit its clinical application. Therefore, efforts have been made to lessen the toxic effects of MMC and improve its utility using various delivery methods.

The dextran-mitomycin $\mathrm{C}$ conjugates synthesized by Hashina et al. and others ${ }^{8-13}$, showed that the conjugates exhibited less cytotoxicity and longer release time than MMC, which prolonged the survival of tumor cells inoculated mice. Based on these results, if the conjugates can further be linked 
with magnetic materials by reversible chemical method, the magnetic conjugates might be led directly to targeted area under the guidance of special magnetic field in vivo or in vitro. The object of this study was to prepare the mitomycin $\mathrm{C}$ with dextran coated magnetic fluids by chemical graft reaction and also investigate its antitumor activities in vivo.

\section{EXPERIMENTAL}

The dextran $70(\mathrm{MW}=70000), 1-[3-($ dimethylamino $)$ propyl]-3-ethylcarbodiimide (EDC.HCl) and 6-bromohexanoic acid were purchased from Sigma; SpectraPor dialysis bag (8000-14000 molecular weight cutoff) was obtained from Rancho Dominguez, USA); Mitomycin C was purchased from Sichuan Antibiotic Factory, China; Sephadex TMG-75 was purchased from Amersham Biosciences, Sweden. Elite flow cytometer was purchased from Couller, America; MTT (from Sigma). All the $\mathrm{FeCl}_{3} \cdot 6 \mathrm{H}_{2} \mathrm{O}, \mathrm{FeCl}_{2} \cdot 4 \mathrm{H}_{2} \mathrm{O}$, hydrochloric acid, sodium hydroxide, etc., were in analytical grade.

Transmission electron microscope (TEM) (JEM-1200EX, SEIKO Electronic Co. Japan); UNICAM UV500 (Thermo Spectronic, England); Nicolet 205 FT-IR (Nicolet, USA); Laser particle analyzer (Malvern Instruments Ltd.); Lyophilizer (LABCONCO Free Zone 12 L); JDM-3 Gaussmeter (product of Jilin University, China); X'Pert Pro X-ray powder diffractometer (XRD, Philips, Holand). RCD-6 dissolution apparatus (Huanghai Drug-detect Factory, Shanghai, China); JDM-13 vibration sample magnetometer (devised by Jilin University, China).

Preparation of magnetic dextran-mitomycin conjugates (MMC-DM) nanoparticles: The process of the preparation of magnetic dextran-mitomycin conjugate was divided into three steps: First, preparation of the dextran magnetic fluids (MF): The mixture solution of $20 \mathrm{~mL}$ of $0.1 \mathrm{M} \mathrm{FeCl}_{3}$ and 10 $\mathrm{mL} \mathrm{FeCl}_{2}$ was added to $42 \mathrm{~mL}$ of $0.2 \mathrm{M} \mathrm{NaOH}$ solution at $60{ }^{\circ} \mathrm{C}$ under agitation, a black precipitation could be found immediately, then adjust $\mathrm{pH}$ to $\mathrm{ca}$. 1.5 and dialyzed them by dialysis bag at $\mathrm{pH} 3.0$, the magnetic fluids contained $\mathrm{Fe}_{3} \mathrm{O}_{4}$ nucleus were acquired. For preparation of the dextran magnetic fluids, the source magnetite sol $40 \mathrm{~mL}$ (contained $\mathrm{Fe}_{3} \mathrm{O}_{4} 21.3 \%$ ) was mixed with $20 \%(\mathrm{w} / \mathrm{v})$ solution of alkali-treated dextran (ATD) $5 \mathrm{~mL}$ and refluxed for $40 \mathrm{~min}$, precipitated by $\mathrm{CH}_{3} \mathrm{OH}$ and dialyzed with running water overnight, the dextran coated $\mathrm{Fe}_{3} \mathrm{O}_{4}$ colloid acquired (abbreviated as DM, as illustrated by conferences $^{4-16}$ in Fig. 1). Second, to synthesize the MMCDM: DM (contained alkali-treated dextran $1 \mathrm{~g}$ ) was dissolved in $20 \mathrm{~mL} \mathrm{NaOH}(4 \mathrm{M}$ ) and 6-bromohexanoic acid $2.6 \mathrm{~g}$ was introduced as spacer into DM at $80^{\circ} \mathrm{C}$ for $6 \mathrm{~h}$ and then cooled to room temperature, dialyzed in running water for $48 \mathrm{~h}$ until the $\mathrm{pH}$ of water outside the dialyze tube reached 6-7 and then the DM with spacer arms (DMS) was obtained. The third: the MMC was coupled to the spacer-introduced DM by the carbodiimide-catalyzed reaction. One gram of 1-ethyl-3-(3dimethylaminopropyl) carbodiimide was added to the solution of spacer-introduced magnetic dextran and the $\mathrm{pH}$ of the solution was controlled at 5.0-5.5 with $1 \mathrm{M} \mathrm{HCl}$. The MMC $(150 \mathrm{mg})$ was dissolved in the above solution and the reaction was carried out at room temperature for $c a .12 \mathrm{~h}$. The product was washed with water, concentrated by ultrafiltration and then

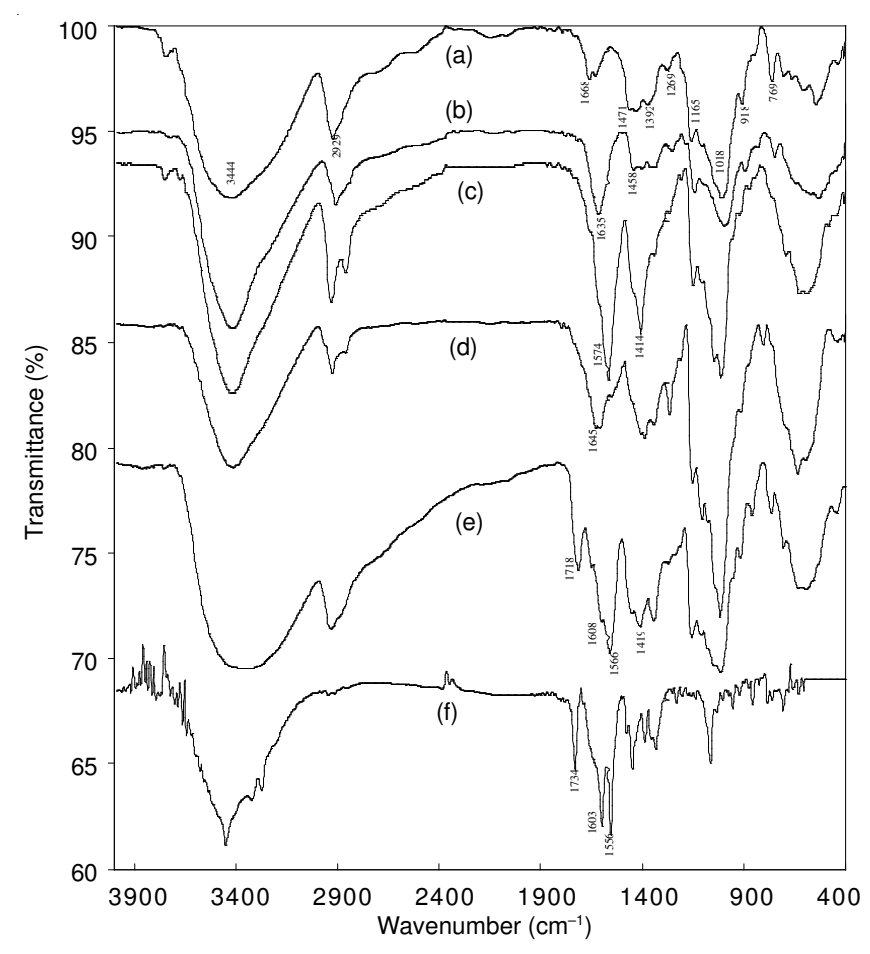

Fig. 1. FT-IR spectra of ATD (a), DM (b), DMS (c), MMC-DM (d), mixture of MMC and DMS (e) and MMC (f)

precipitated by acetone to yield purple solid. The compounds were prepared in saline solution at an adequate concentration for injection. The whole process of MMC and DM reaction was kept away from light.

Characterization of MMC-DM: The XRD analysis was performed on the $\mathrm{X}^{\prime}$ Pert Pro $\left(\mathrm{CuK}_{\alpha}, \lambda=1.5418 \AA\right.$, scan rate is $4 \%$ min). The MF, DM and MMC-DM nanoparticles were respectively dried and crushed into powders as samples. The standard peak was recorded under the same conditions.

IR spectra: ATD, DM, DMS, mixture of MMC and DMS, MMC-DM and pure MMC were separately dried and mixed with potassium bromide and then compressed into a thin pellet on a Nicolet 205 Fourie transform infrared spectrometer.

The diameter and size distribution of the prepared conjugate nanoparticles were measured at a Malvern 3000 HAS Zeta sizer using the PCS and ELS analysis, respectively. The experiments were carried out at $25^{\circ} \mathrm{C}$ and distilled water was used as dispersant solution. For zeta potential measurement, the MMC-DM was diluted to obtain a concentration of $20 \mathrm{mg}$ $\mathrm{Fe}_{3} \mathrm{O}_{4} / \mathrm{mL}$ with the Britton-Robins in buffer solutions at $\mathrm{pH}$ 6.5. The TEM of the MMC-DM nanoparticles microphotographs were observed at JEM-1200EX. To obtain the samples for TEM observations, one drop of MMC-DM nanoparticles aqua dispersion suspension was placed on a copper grid covered with nitrocellulose membrane and nitrogen gas-dried at $25^{\circ} \mathrm{C}$ before negative staining with $1 \%$ phosphotungstic sodium solution. The saturation magnetization of the MMC-DM was measured at a JDM-13 vibration sample magnetometer at room temperature with maximum applied field of $1.8 \mathrm{~T}$.

Grafting ratio and drug loading content: The grafting ratio of the MMC linked to DMS and the drug loading content were determined by eqns. 1 and 2 . To determine the grafting ratio and the drug loading content, gel filtration chromatography 
(Sephadex G-75) method was adopted (the parameters of packed column were: diameter: $1 \mathrm{~cm}$; length: $25 \mathrm{~cm}$; flow rate: $0.3 \mathrm{~mL} / \mathrm{min}$, temperature: $25^{\circ} \mathrm{C}$ ). The parts of free MMC could be assembled based on the intrinsic colour and detected by the ultraviolet absorption at maximum absorption wavelengh of MMC in $365 \mathrm{~nm}$.

Grafting ratio $(\%)$

$$
=\frac{\text { weight of the MMC in nanopartides }}{\text { weight of the feeding MMC }} \times 100 \%
$$

Drug loading content (\%)

$$
=\frac{\text { weight of the MMC in nanopartides }}{\text { weight of the nanopartides }} \times 100 \%
$$

In vitro evaluation of cell toxic by MTT assay: MTT (Sigma) was dissolved in sterile phosphate-buffered saline (PBS) at a concentration of $5 \mathrm{mg} / \mathrm{mL}$ and filtered through 0.22 $\mathrm{nm}$ filter. Subcultured L-02 cell was centrifuged to concentration $1 \times 10^{7} / \mathrm{L}$, cultured in 96 well microplates in $100 \mu \mathrm{L}$ $\left(1 \times 10^{5} /\right.$ well $)$ which were added with varies kind concentration of MMC, MMC-MD, MD and blank solution. Following incubation at $37^{\circ} \mathrm{C}$ for $0.5 \mathrm{~h}$ in $5 \% \mathrm{CO}_{2} / 95 \%$ air humidified atmosphere, the reduced MTT was observed by light microscopy within PBMC (MTT staining).

The spectrophotometric measurement of the formazan was taken by prolonging the incubation period to $3 \mathrm{~h}$. The plates were then centrifuged at $1000 \mathrm{rpm}$ for $3 \mathrm{~min}$, the supernatants decanted to remove the untransformed MTT and $150 \mu \mathrm{L}$ of dimethyl sulphoxide (DMSO) added to each well. Purple crystals of formazan were dissolved by vigorously shaking microplates and absorbance was measured using a microplate spectrophotometer at wavelength $570 \mathrm{~nm}$. Results were expressed as absorbance (A). Reported values are the relative growth rate $(\mathrm{RGR} \%=$ experiment group/control group $\times$ $100 \%$ ) listed in Table-1.

In vitro release of the MMC from MMC-DM: In vitro release of MMC from the MMC-DM was evaluated using a dialysis bag diffusion technique. A phosphate-buffered saline (PBS, pH 7.4) solution contained $0.1 \%$ sodium azide to prevent decomposition was adopted as release media. The general process was as follows: Six portions of the lyophilized samples were weighted out exactly (drug loading content was $8.53 \%$, each weight was $58.50 \mathrm{mg}$ ) and packed into PBS soaked dialysis bags, then hung them immediately into dissolution bottles that contained $300 \mathrm{~mL}$ PBS each at the temperature of $37 \pm 1{ }^{\circ} \mathrm{C}$ and stirred simultaneously at speed of 100 $\mathrm{rpm} / \mathrm{min}$, took out $5 \mathrm{~mL}$ of the dissolution at each specified time and added immediately the same volume of blank PBS solution into the bottles. The samples were detected at 365 $\mathrm{nm}$ on ultraviolet spectrophotometry detection. The whole process was operated under shade condition. The pure MMC sample was also operated at the same process.

In vivo antitumor activity: $\mathrm{BACA} / \mathrm{C}$ nude mice weighing 18-20 g were subcutaneously inoculated with Bel-7402 human liver tumor cells. After 10 days, growth prosperity tumor tissue was chosen to cut into pieces to $c a .2 \mathrm{~mm}^{3}$ to inoculate each to nude mice, respectively. The process was manipulated under the condition of $25-27^{\circ} \mathrm{C}$, relative humidity $40-60 \%$ and specific-pathogen free (SPF) condition. When the average volumes of the subcutaneous tumor grow to 100$300 \mathrm{~mm}^{3}$, the animals were divided into 6 groups and 5 mice each for injection of chemotherapy. The magnetic masses with $4500 \mathrm{Gs}$ were fixed on the skin of the tumor by adhesive tape.

The administration was carried out by $i . v$. in the tail vein injection of mice. The first three groups were injected MMCDM physiological saline solution with a dosage of (MMC) of $0.75 \mathrm{mg} / \mathrm{kg}$ (low dosage), $1.5 \mathrm{mg} / \mathrm{kg}$ (middle dosage) and 3.0 $\mathrm{mg} / \mathrm{kg}$ (high dosage), respectively under magnetic field for 40 min. As comparison, the $4^{\text {th }}$ to $6^{\text {th }}$ groups were injected with MMC-DM physiological saline solution with the dosage of $1.5 \mathrm{mg} / \mathrm{kg}$ (middle dosage), pure MMC injectable powder in physiological saline solution with a dosage of $1.5 \mathrm{mg} / \mathrm{kg}$ and pure physiological saline (PS) solution, respectively under no magnetic fluid condition. The administrations were repeated every 2 days for 2 weeks. At each time the animals were weighed and the volumes of the tumor were measured in vitro by sliding caliper before administration. After 2 weeks, the mice were executed and peeled to take out the tumors and then weighed and measured the ex vivo tumor volume.

The pharmacodynamic results of the MMC-DM samples could be expressed by the percentage of the weight and volume tumor inhibiting rate (TIR \%). The weight of the TIR can be calculated as: TIR $(\mathrm{W}) \%=(1-\mathrm{T} / \mathrm{C}) \times 100 \%$ where $\mathrm{T}$ and $\mathrm{C}$ represent the mean weight tumor of treated animal and that of the control animal, respectively.

The volume of tumor can be calculated as $\mathrm{V}=\left(\mathrm{L} \times \mathrm{W}^{2}\right) /$ 2 , where the $\mathrm{L}$ represent the long diameter of tumor and $\mathrm{W}$ represent the short diameter. The volume TIR can be expressed as:

$$
\operatorname{TIR}(\mathrm{V})=\left(\frac{1-\mathrm{DV}_{\text {treated group }}}{\mathrm{DV}_{\text {Control group }}}\right) \times 100 \%
$$

The results were analyzed by one way analysis of variance (ANOVA), followed by the paired P value by SPSS 13.0.

\begin{tabular}{|c|c|c|c|c|c|c|}
\hline \multirow{2}{*}{$\mathrm{MMC}(\mathrm{mg} / \mathrm{L})$} & \multicolumn{2}{|c|}{ MMC } & \multicolumn{2}{|c|}{$\mathrm{DM}$} & \multicolumn{2}{|c|}{ MMC-DM } \\
\hline & A & RGR (\%) & A & RGR (\%) & $\mathrm{A}$ & RGR (\%) \\
\hline 0.4 & $0.240 \pm 0.006^{*}$ & 79.0 & $0.290 \pm 0.004 *$ & 95.4 & $0.273 \pm 0.004 * *$ & 90.00 \\
\hline 0.8 & $0.202 \pm 0.003^{*}$ & 66.4 & $0.286 \pm 0.003^{*}$ & 94.2 & $0.259 \pm 0.003 * *$ & 85.40 \\
\hline 1.2 & $0.155 \pm 0.003^{*}$ & 51.3 & $0.285 \pm 0.003 *$ & 94.0 & $0.226 \pm 0.002 * *$ & 74.30 \\
\hline 1.6 & $0.101 \pm 0.002 *$ & 33.2 & $0.281 \pm 0.003^{*}$ & 92.1 & $0.210 \pm 0.003 * *$ & 69.20 \\
\hline 2.0 & $0.061 \pm 0.001 *$ & 20.2 & $0.274 \pm 0.005^{*}$ & 90.3 & $0.188 \pm 0.004 * *$ & 62.10 \\
\hline 2.4 & $0.043 \pm 0.001 *$ & 14.3 & $0.268 \pm 0.002 *$ & 88.3 & $0.174 \pm 0.001 * *$ & 57.40 \\
\hline
\end{tabular}

TABLE-1

EFFECT OF MMC, MMC-DM, DM NANOPARTICLES ON L-02 CELL GROWTH ( $\mathrm{n}=4$ ) 
The pathological sections were also observed to verify the effects of MMC-DM on tumor and normal tissues. The samples gathered were washed in phosphate buffer saline and then fixed in $4 \%$ neutral buffered formalin before histological analyses and sectioned at $5 \mathrm{~mm}$ thickness, stained with hematoxylin and eosin (H\&E), evaluated on OLYMPUS CK40 light microscope.

\section{RESULTS AND DISCUSSION}

Synthesis of the dextran magnetic (DM): The synthesis of dextran-magnetic was referred to the articles ${ }^{14,15,17}$. The process of the reaction could be illustrated as Fig. 2 according to the illustration of these articles. Fig. 1 illustrated the FT-IR spectra of alkali treated dextran (ATD) (a), DM (b), DMS (c), MMC-DM (d), mixture of MMC and DMS (e) and MMC (f). The band near to $1108 \mathrm{~cm}^{-1}$ was assigned to the vibration of the $\mathrm{C}-\mathrm{O}$ bond at the $\mathrm{C}-4$ position of the glucose residue ${ }^{18}$. Two peaks at $\bar{v}=1668$ and $1471 \mathrm{~cm}^{-1}$ were shown in Fig. (a), which were the two characteristic peaks for identifying a carboxylic salt, were attributed by the asymmetric and symmetric stretching vibration of the $\mathrm{COO}^{-19-23}$, but in Fig. (b), the two peaks exhibited red shift to 1635 and $1458 \mathrm{~cm}^{-1}$, respectively, The little difference in FT-IR spectra of ATD and DM was explained ${ }^{24}$ that there might had intermolecular interactions occurrence between the dextran and magnetic fluid, which could keep the relative stability of the DM effectively.

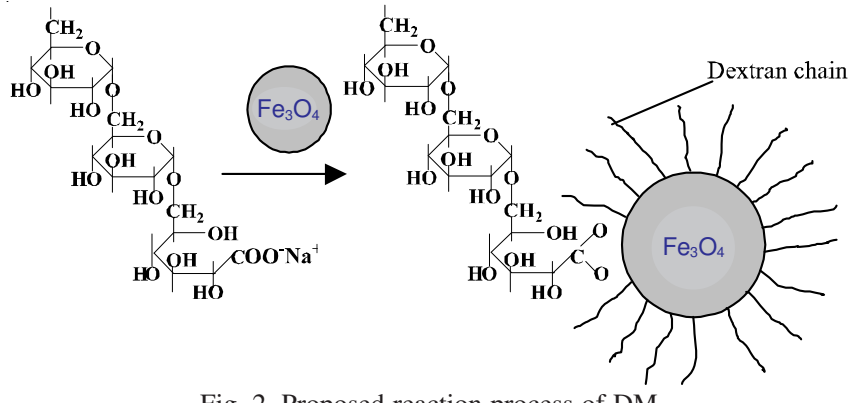

Fig. 2. Proposed reaction process of DM

The two strong peaks at 1574 and $1414 \mathrm{~cm}^{-1}$ were assigned to the asymmetric and symmetric stretching vibration of the $\mathrm{COO}^{-}$at the terminal group of the spacer arm linked to the $\mathrm{DM}$ as shown in (c). The peak at $1108 \mathrm{~cm}^{-1}$ was assigned to stretching of ether linkages (C-O-C). Compared with the IR spectra of (c), (d) and (e), the characteristic peaks of carboxylic salt of spacer disappeared but a new peak at $1645 \mathrm{~cm}^{-1}$ appeared in (d), which indicated the presence of imine bonds ${ }^{7}$. The peak was not found in the FT-IR spectra of the mixture of DMS and MMC or the MMC itself, which also indirectly implied that chemical reaction might occur between MMC and DMS.

Synthesis of MMC-DM: In the process of synthesis of MMC-DM, the dextran in the DM surface was activated by alkali, 6-bromohexanoic acid was introduced as a spacer, MMC was conjugated to spacer-introduced dextran by carbodiimidecatalyzed reaction. The proposed structure ${ }^{10,12,13,25}$ can be illustrated as Fig. 3 .

Particle size distribution and morphology: The typical TEM image of MMC-DM nanoparticles was shown in Fig. 4. It was found that the nanoparticles had a near sphere shape

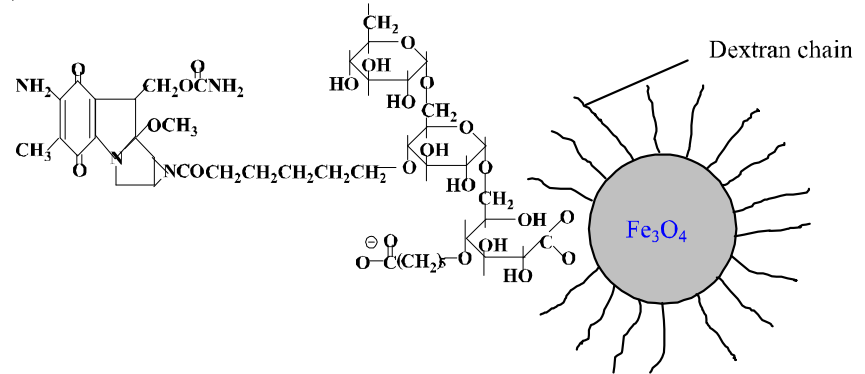

Fig. 3. Proposed chemical structure of MMC-DM

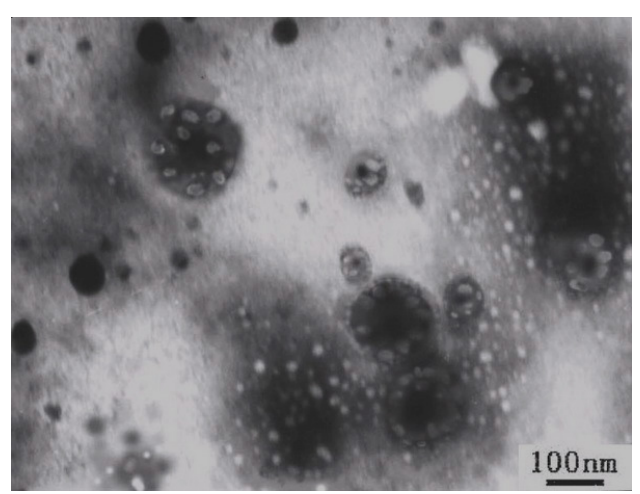

(a)

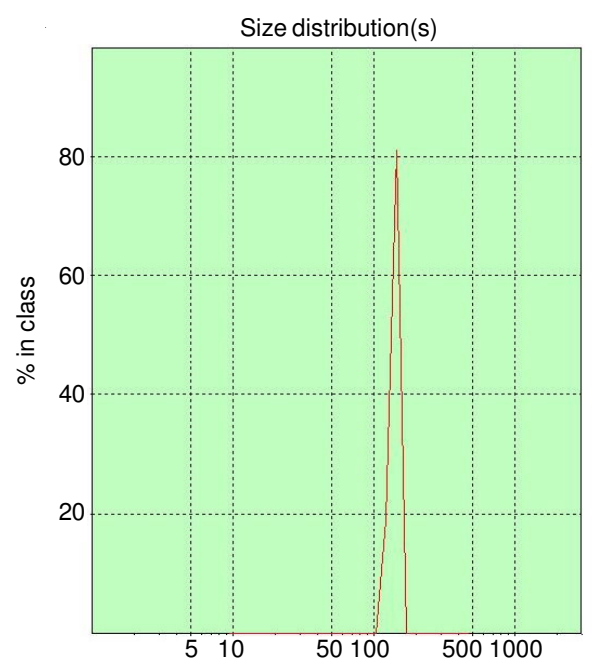

(b)

Fig. 4. Typical TEM image (a) and distribution (b) of MMC-DM

and each nanoparticle contained some smaller particles which could be considered to be the aggregation of $\mathrm{Fe}_{3} \mathrm{O}_{4}$ nanoparticles and the average diameter of the MMC-DM and magnetic fluid encapsulated in the nanoparticles was $c a .125$ and $11 \mathrm{~nm}$, respectively. The size of the magnetic fluid core less than $13 \mathrm{~nm}$ in the conjugate was considered to have supermagnetic property ${ }^{26}$. Additionally, there might be some nanoparticles adherence to the nitrocellulose membrane on the copper grid which could lead the particles collapse during the drying process and dispersed in some directions, resulting in an irregular shape of some nanoparticles.

Size distribution and zeta potential may play important roles in determining the fate of nanoparticles after administration ${ }^{27}$. Fig. 4 showed the representative particle size distribution. As is shown, the mean diameter of the nanoparticles was $c a .125 \mathrm{~nm}$, which was consisted with the result of the 
TEM image and the low polydispersion index (average 0.23 ) indicated the prepared nanoparticles have relatively uniform diameter and narrow distribution.

The mean Zeta potential of MMC-DM nanoparticles in this experiment was $c a$. $-26.60 \mathrm{mV}$. Due to the frequency of the gaps of liver fenestrate larger than $200 \mathrm{~nm}$ is less than $1 \%{ }^{28}$, the larger particles are difficult to penetrate through the liver's parenchymal cell. Smaller particles were also tended to accumulate in the tumor sites due to the EPR (enhanced permeability and retention) effect ${ }^{29}$. The progressive accumulation of the particles in Kupffer cells can result in a negative enhancement $^{30}$. So, this negative charged surface of the nanoparticles from the naked carboxyl $\left(-\mathrm{COO}^{-}\right)$groups can meet the need of uptake of the liver target.

XRD analysis: Due to the preparation of MMC-DM nanoparticles must experience several steps of chemical reaction, in which many chemical reagents, such as acid and alkali substances participated in the process, the structure of $\mathrm{Fe}_{3} \mathrm{O}_{4}$ in the nanoparticles might be destroyed at any step. To keep steady and unchanged structure of the $\mathrm{Fe}_{3} \mathrm{O}_{4}$ is crucial in ensuring the superparamagnetis. The XRD analysis is usually used to verify the structure of the $\mathrm{Fe}_{3} \mathrm{O}_{4}$. The spectrums of the resultant magnetic fluid (MF), DM and MMC-DM nanoparticles were shown in Fig. 5, several characteristic peaks for $\mathrm{Fe}_{3} \mathrm{O}_{4}\left(2 \theta=30.39^{\circ}, 35.81^{\circ}, 43.53^{\circ}, 57.59^{\circ}, 63.25^{\circ}\right)$, marked by their indices ((220), (311), (400), (511), (440)) could be observed. The character of the broadened XRD peaks of the magnetic nanoparticle was consistent with standard pattern of $\mathrm{Fe}_{3} \mathrm{O}_{4}$. The broadening of the peaks were probably attributed to the matrix constrains of the nanosized particles. The average grain size of $\mathrm{Fe}_{3} \mathrm{O}_{4}$ nanocrystal could be calculated by Scherer formula: $D_{\text {hkl }}=K \lambda / B_{1 / 2} \cos \theta$. Little difference among diffraction peak of the MF, DM and MMC-DM indicated that the structure of $\mathrm{Fe}_{3} \mathrm{O}_{4}$ nucleus could kept unchanged after serious chemical reactions.

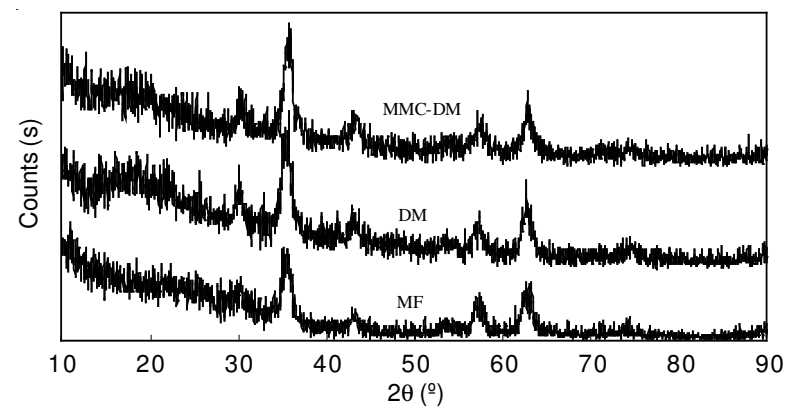

Fig. 5. XRD diagrams of MMC-DM

Magnetic properties: The magnetic properties were detected using a JDM-13 vibration sample magnetometer. Fig. 6 showed the result of the MMC-DM (content of $\mathrm{Fe}_{3} \mathrm{O}_{4}$ was $27.0 \%$, drug loading was $8.53 \%$ ) under temperature 293.0 $\mathrm{K}$ and field intensity $5 \mathrm{kOe}$ condition. The saturation magnetization was $0.57 \mathrm{emu} / \mathrm{g}$ and no hysteresisloop observed. The values of residual magnetization $(\mathrm{Mr})$ and coercivity $(\mathrm{Hc})$ were zero indicated that the MMC-DM owned superparamagnetic behaviour, which ensured good magnetic oriented behaviour produced by $\mathrm{Fe}_{3} \mathrm{O}_{4}$ particles in the MMC-DM under magnetic field ${ }^{26}$.

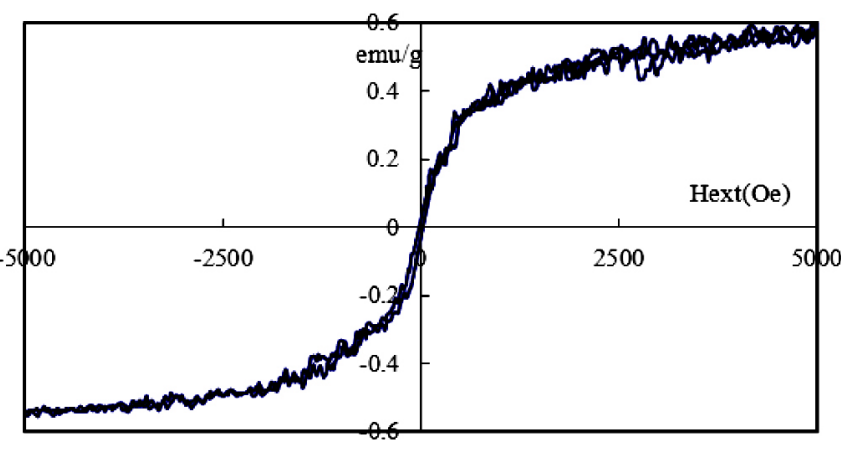

Fig. 6. Magnetization of MMC-DM

Drug loading and grafting ratio: An ideal nanoparticle drug with highest drug loading and highest entrapment efficiency can decrease the quantity of carrier required but afford sufficient amount of active compound (drug) to the target site ${ }^{31}$. In this experiment, three batches of MMC-DM samples were prepared according to the method mentioned before. The conjugation of MMC to DM was confirmed by gel filtration. The results showed the average grafting ratio of MMC to DM was $89.8 \%$ and the drug loading was $c a .8 .53 \%$.

MTT assay: Table- 1 revealed that cell toxic of the DM was very low compared with the blank group and the MMCMD showed relatively lower toxic to L-02 cell compared with MMC. The result suggested that the MMC conjugated MD lessened the toxic of MMC to cells.

In vitro release study: Fig. 7 illustrated the cumulative release profiles of pure MMC and MMC from MMC-DM nanoparticles at $37 \pm 1^{\circ} \mathrm{C}$ in $\mathrm{pH} 7.4 \mathrm{PBS}$. It can be seen that the MMC from the MMC-DM sample release reached to $17.5 \%$ at the early $2 \mathrm{~h}$, which meant a burst release. After a fast release of $c a .4 \mathrm{~h}$, the release rate of MMC from MMC$\mathrm{DM}$ inclined to slow and became steady. In the case of this study, ca. $57 \%$ of the MMC from MMC-DM released in $48 \mathrm{~h}$, which suggested that there were still parts of MMC could not release in the experiment time. As comparison, pure MMC could be released from the dialysis bags quickly and all MMC released almost completely within $2 \mathrm{~h}$.

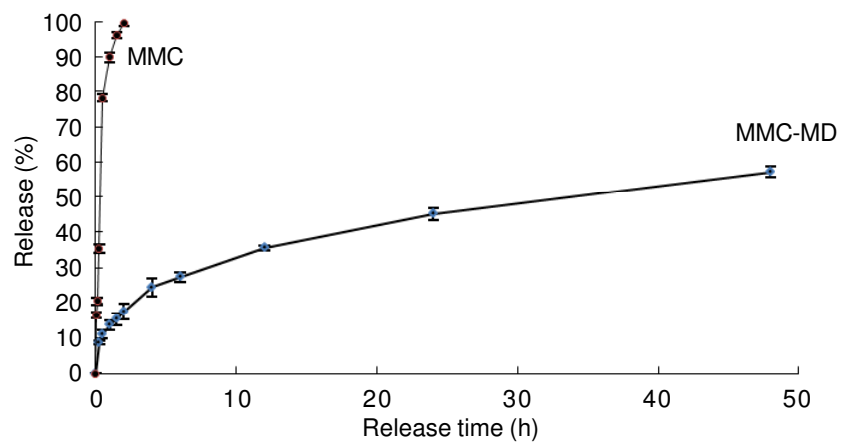

Fig. 7. Cumulative in vitro release profiles of MMC-DM nanoparticles and $\mathrm{MMC}$

The in vitro release of MMC from MMC-DM depend on hydrolyze speed of acid amide bond. The behaviour of the in vitro release of MMC from MMC-DM might attribute to following reasons: The hydrolyzation of acid amide might experience a relatively long time at physiology condition and there might have some bonded MMC-DM exists in the inner 
MMC-DM nanoparticles, which need time to go through the core cell after MMC liberated from MMC-DM ${ }^{32}$.

In vivo antitumor activity: Table- 2 showed that there were significant differences compared the weight of the tumor of treated group under magnetic field and the middle dosage MMC-DM group $(1.5 \mathrm{mg} / \mathrm{kg})$ without magnetic field with the pure MMC and PS group $(p<0.01)$, which indicated the weight of tumor of the treated group under magnetic field was obviously lower than the MMC or PS group. There were also significant differences between the middle dosage group with magnetic field and without magnetic field $(p=0.028<0.05)$. The result revealed that the administrator under the exterior magnetic field exhibited stronger inhibiting properties than under normal condition, which could also be deduced that the exterior magnetic field could lead the magnetic nanoparticles drug release in targeted area. The similar results could be summarized from the volume TIR of Table-3.

TABLE-2

WEIGHT AND THE TIR(W) \% OF THE TUMOR

\begin{tabular}{ccccc}
\hline Group & $\begin{array}{c}\text { Weight of tumor } \\
(\mathrm{g})\end{array}$ & $\begin{array}{c}\text { TIR(W) } \\
(\%)\end{array}$ & $\begin{array}{c}p \text { value } \\
(\text { with PS) }\end{array}$ & $\begin{array}{c}p \text { value (with } \\
\text { MMC) }\end{array}$ \\
\hline L + M & $0.1564 \pm 0.0372$ & 69.96 & 0 & 0 \\
M + M & $0.0880 \pm 0.0141$ & 83.1 & 0 & 0 \\
H + M & $0.0518 \pm 0.0123$ & 90.05 & 0 & 0 \\
M & $0.2058 \pm 0.0564$ & 60.46 & 0 & 0.012 \\
MMC & $0.3146 \pm 0.0198$ & 39.57 & 0 & - \\
PS & $0.5206 \pm 0.0820$ & - & - & 0 \\
\hline
\end{tabular}

Note: The symbol L, MD, HD, M, PS represent the low dosage, middle dosage, high dosage, Magnetic field, physiological saline respectively.

\begin{tabular}{ccccc}
\multicolumn{5}{c}{ TABLE-3 } \\
\multicolumn{5}{c}{ VALUE OF NET VOLUME INCREASED } \\
AND TIR(V) \% OF THE TUMOR \\
\hline Group & $\begin{array}{c}\Delta \text { V of value } \\
\left(\mathrm{mm}^{3}\right)\end{array}$ & $\begin{array}{c}\text { TIR(V) } \\
(\%)\end{array}$ & $\begin{array}{c}p \text { value } \\
(\text { with PS })\end{array}$ & $\begin{array}{c}p \text { value (with } \\
\text { MMC) }\end{array}$ \\
\hline L + M & $0.1451 \pm 0.0218$ & 71.29 & 0 & 0.016 \\
M + M & $0.0861 \pm 0.0056$ & 82.96 & 0 & 0.001 \\
H + M & $0.0410 \pm 0.0030$ & 91.89 & 0 & 0 \\
M & $0.1722 \pm 0.0297$ & 65.94 & 0 & 0.045 \\
MMC & $0.2942 \pm 0.0220$ & 41.8 & 0.001 & - \\
PS & $0.5055 \pm 0.2184$ & - & - & 0.001 \\
\hline
\end{tabular}

Figs. 8 and 9 illustrate the pathological photos of high dosage treated group $(3.0 \mathrm{mg} / \mathrm{kg})$ and MMC treated group originate from tumor and liver, respectively. The tumor of high dosage treated group under the leading of ex vivo magnetic field showed severe cellular necrosis and the number of caryocinesia declined (as sparrow pointed) than MMC group. The liver pathological photos showed that there were damages happened in the MMC group. The results suggested that the MMC in the MMC-DM conjugate nanoparticles could be released on targeted sites and thus could avoid chemotherapeutics to normal issues.

\section{Conclusion}

Mitomycin C linked dextran magnetic fluid (MMC-DM) conjugate nanoparticles were prepared successfully by carbodiimide-catalyzed reaction. The in vitro release character and in vivo antitumor in rats were also investigated. The results showed a high grafting ratio and a high loading capacity

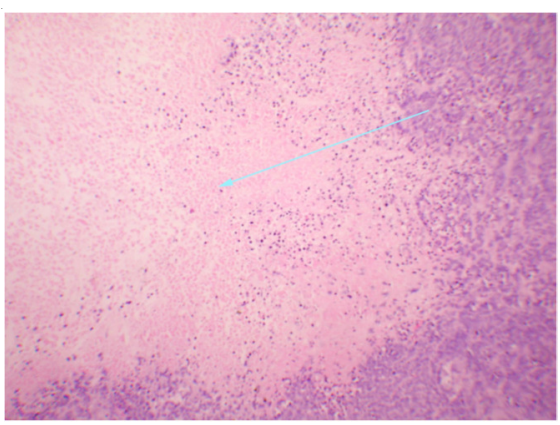

(a)

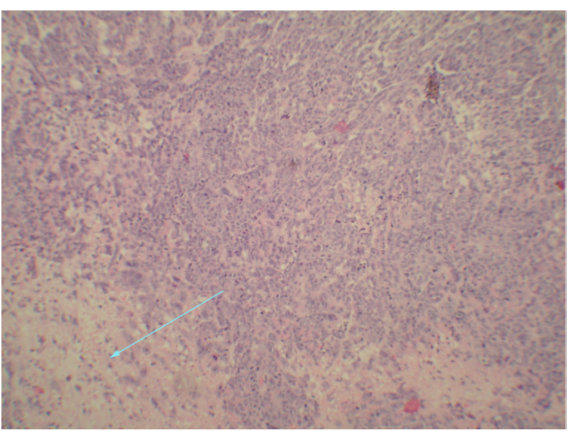

(b)

Fig. 8. Tumor pathological photos of high dosage treated group (a) and MMC treated group (b)

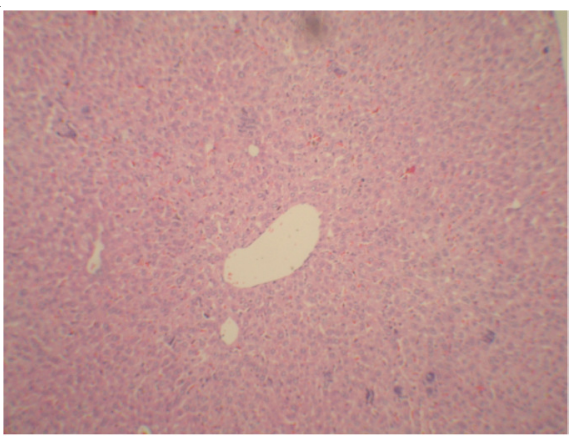

(c)

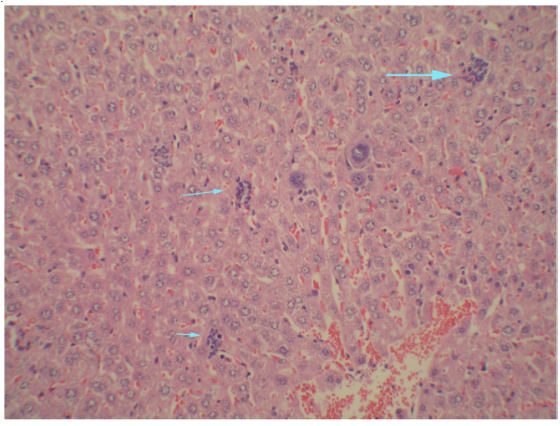

(d)

Fig. 9. Hepar pathological photos of high dosage treated group (c) and MMC treated group (d)

of magnetic conjugate naoparticles could be obtained by our method. The MMC in the MMC-DM in vitro release exhibited extended release, which also revealed the drug could be released effectively from the conjugate. MMT assay indicated that the conjugate had a low cell toxic than the MMC alone. By the help of ex vivo magnetic field, the in vivo antitumor in rats exhibited a site targeted effect. 


\section{ACKNOWLEDGEMENTS}

The authors thank Assoc. Prof. Guo Cong Lin, Physical Department, Zhongshan Universiy, China for his kind help in the characterization of magnetic property. The work was supported by China Postdoctoral Science Fund (20080430856) and High educated talents fund of Xinxiang Medical University, China (100778).

\section{REFERENCES}

1. D.R. Friend and S. Pangburn, Med. Res. Rev., 7, 53 (1987)

2. R.M. Mainardes and L.P. Silva, Curr. Drug Targets, 5, 449 (2004)

3. M. Thanou and R. Duncan, Curr. Opin. Investig. Drugs, 4, 701 (2003).

4. M.E. Davis, Mol. Pharm., 6, 659 (2009).

5. R. Duncan and F. Spreafico, Clin. Pharmacokinet., 27, 290 (1994).

6. S.S. Dhaneshwar, M. Kandpal, N. Gairola and S.S. Kadam, Indian J. Pharm. Sci., 68, 705 (2006).

7. R.Y. Cheung, Y. Ying, A.M. Rauth, N. Marcon and W.X. Yu, Biomaterials, 26, 5375 (2005)

8. S.K. Nune, P. Gunda, B.K. Majeti, P.K. Thallapally and M.L. Forrest, Adv. Drug Deliv. Rev., 63, 876 (2011).

9. M. Hashina, Y. Takakura, S. Matsumoto, H. Sasaki, A. Kato, T. Kojima, S. Muranishi and H. Sezaki, Chem. Pharm., Bull. (Tokyo), 31, 2055 (1983).

10. Y. Takakura, M. Kitajima, S. Matsumoto, M. Hashida and H. Sezaki, Int. J. Pharm., 37, 135 (1987).

11. Y. Takakura, R. Atsumi, M. Hashida and H. Sezaki, Int. J. Pharm., 37, 145 (1987).

12. T. Nomura, A. Saikawa, S. Morita, T. Sakaeda (ne Kakutani), F. Yamashita, K. Honda, Y. Takakura and M. Hashida, J. Control. Rel., 52, 239 (1998).

13. A. Noguchi, T. Takahashi, T. Yamaguchi, K. Kitamura, Y. Takakura, M. Hashida and H. Sezaki, Bioconjugate Chem., 3, 132 (1992).
14. T. Kawaguchi and M. Hasegawa, J. Mater. Sci.: Mater. Med., 11, 31 (2000).

15. T. Kawaguchi, T. Hanaichi, M. Hasegawa and S. Maruno, J. Mater. Sci.: Mater. Med., 12, 121 (2001).

16. M. Hasegawa, T. Hanaichi, H. Shoji, T. Kawaguchi and S. Maruno, J. Appl. Phys. (Japan), 37, 1029 (1998).

17. L. Guo, G. Liu, R.-Y. Hong and H.Z. Li,Mar. Drugs, 8, 2212 (2010).

18. K.I. Shingel, Carbohydr. Res., 337, 1445 (2002).

19. M. Wen, Q.-Y. Liu, Y.-F. Wang, Y.-Z. Zhu and Q.-S. Wu, Colloid. Surf. A, 318, 238 (2008).

20. S. Liu, X. Wei, M. Chu, J. Peng and Y. Xu, Colloid. Surf. B, 51, 101 (2006).

21. J. Li, Y. Zhu, J. Du, J. Zhang and Y. Qian, Solid State Commun., 147, 36 (2008).

22. S. Chena and W. Liu, Mater. Chem. Phys., 98, 183 (2006).

23. Y. Kim, P. Kim, C. Kim and J. Yi, Korean J. Chem. Eng., 22, 321 (2005).

24. X.Q. Xu, H. Shen, J.R. Xu, J. Xu, X.J. Li and X.M. Xiong, Appl. Surf. Sci., 252, 494 (2005).

25. S. Matsumoto, A. Yamamoto, Y. Takakura, M. Hashida, N. Tanigawa and H. Sezaki, Cancer Res., 46, 4463 (1986).

26. N.A.D. Burke, H.D.H. Stöver and F.P. Dawson, Chem. Mater., 14, 4752 (2002).

27. Y. Dong and S.S. Feng, Biomaterials, 25, 2843 (2004).

28. E. Wisse, R.B. De Zancer, K. Charels, P. Van Der Smissen and R.S. McCuskey, Hepatology, 5, 683 (1985).

29. F. Yuan, M. Leunig, S.K. Huang, D.A. Berk, D. Papahadjopoulos and R.K. Jain, Cancer Res., 54, 3352 (1994).

30. P. Oswald, O. Clement, C. Chambon, E. Schouman-Claeys and G. Frija, Magnetic Resonance Imaging, 15, 1025 (1997).

31. T. Govender, S. Stolnik, M.C. Garnett, L. Illum and S.S. Davis, J. Control. Rel., 57, 171 (1999).

32. C. Allen, D. Maysinger and A. Eisenberg, Colloid. Surf. B, 16, 3 (1999). 